\title{
TEOS: A framework for constructing operational definitions of medication adherence based on Timelines - Events - Objectives - Sources
}

\author{
Alexandra L. Dima*, PhD, Health Services and Performance Research (HESPER EA 7425), \\ University Claude Bernard Lyon 1, Lyon, France; alexandra.dima@univ-lyon1.fr \\ Samuel S. Allemann*, PhD, Pharmaceutical Care Research Group, University of Basel, Basel, \\ Switzerland \\ Jacqueline Dunbar-Jacob, PhD, University of Pittsburgh School of Nursing, Pittsburgh, PA, \\ USA \\ Dyfrig A. Hughes, PhD, Centre for Health Economics and Medicines Evaluation, Bangor \\ University, Bangor, North Wales, United Kingdom \\ Bernard Vrijens $^{\#}$, PhD, AARDEX Group \& Department of Public Health Liège University, \\ Liège, Belgium \\ Ira B. Wilson", PhD, Department of Health Services, Policy, and Practice, Brown University \\ School of Public Health, Providence, RI, USA \\ * Drs. Dima and Allemann share joint first authorship. \\ ${ }^{*}$ Drs. Wilson and Vrijens share joint last authorship.
}

\begin{abstract}
Objectives: Managing adherence to medications is a priority for health systems worldwide. Adherence research is accumulating, yet the quality of the evidence is reduced by various methodological limitations. In particular, the heterogeneity and low accuracy of adherence measures have been highlighted in many literature reviews. Recent consensus-based guidelines advise on best practices in defining adherence $(\mathrm{ABC})$ and reporting of empirical studies (EMERGE). While these guidelines highlight the importance of operational definitions in adherence measurement; such definitions are rarely included in study reports. To support researchers in their measurement decisions, we developed a structured approach to formulate operational definitions of adherence.

Methods: A group of adherence and research methodology experts used theoretical, methodological and practical considerations to examine the process of applying adherence definitions to various research settings, questions and data sources. Consensus was reached through iterative reviewing of discussion summaries and framework versions.

Results: We introduce TEOS, a four-component framework to guide the operationalization of adherence concepts: 1) describe treatment as four simultaneous interdependent timelines (recommended and actual use, conditional on prescribing and dispensing); 2) locate four key events along these timelines to delimit the three ABC phases (first and last recommended use, first and last actual use); 3) revisit study objectives and design to finetune research questions and assess measurement validity and reliability needs, and 4) select data sources (e.g., electronic monitoring, self-report, electronic healthcare databases) that best address measurement needs.
\end{abstract}

Conclusion: Using the TEOS framework when designing research and reporting explicitly on these components can improve measurement quality.

Keywords: medication adherence; persistence; measurement; self-report; electronic healthcare data; electronic monitoring 


\section{Introduction}

Research on medication adherence has developed substantially in recent years. However, evidence synthesis is constrained by considerable heterogeneity in study results, which is determined largely by the low quality of measurement and high heterogeneity in adherence measures. ${ }^{1}$ So far, various methods for measurement have been used in research and clinical practice, many with limited theoretical grounding and little or no validation. ${ }^{2}$ Literature reviews report large differences in estimates of the prevalence of non-adherence and its relationships with determinants and outcomes; $;^{3-5}$ and weak associations between measures, even in the same populations. ${ }^{6,7}$ Improvement of adherence measurement is therefore a priority for the field.

Several guidelines and recommendations have been proposed to improve the precision and standardization in adherence definitions and measurement. The consensus-based theoretical framework developed within the $\mathrm{ABC}$ (Ascertaining Barriers to Compliance) project $^{8}$ provided conceptual definitions for adherence and terminology that have been widely adopted in adherence research. $\mathrm{ABC}$ distinguished between three phases $^{1}$ : initiation (starting treatment), implementation (following the treatment as prescribed), and discontinuation (stopping treatment earlier than recommended). It also highlighted the need to measure each independently and investigate specific causes and consequences for effective adherence management. Several literature reviews have proposed recommendations on adherence measurement and its three phases either by comparing different data sources ${ }^{2,9-11}$ or by reviewing alternative methods using the same type of data source. ${ }^{7,12}$ They converge towards the view that there is no single, 'gold standard' measure for the overarching concept of medication adherence. Instead, optimal measurement needs to consider numerous theoretical, methodological and practical aspects and find a balance between generalizability and adaptation to the specific needs of individual studies.

\footnotetext{
${ }^{1}$ The word "phases" is appropriate because of the natural, consecutive order of starting, implementing, and stopping a medication. But because a medication may be started and stopped multiple times, the word "component" may in some cases be more useful.
} 
Guidelines for reporting adherence research have been proposed recently. For clinical trials, an outcome specification format has been suggested which requires information on four levels: domain, specific measurement, specific metric, and aggregation method. ${ }^{13}$ The EMERGE guidelines ${ }^{14}$ translated this general format for adherence research and recommended specification of the adherence phase(s), operational definitions, measurement methods including metric and statistical details, and summary results. However, while conceptual definitions and measures are commonly reported in empirical studies, operational definitions are rarely detailed. In this paper we propose practical recommendations we believe will facilitate the development and description of operational definitions.

\section{Methods}

Following the development of the EMERGE guidelines, an adherence methodology working group was convened, comprising of six experts in adherence measurement (each a member of ESPACOMP, four also members of the EMERGE steering committee). The working group collaborated between November 2016 and November 2019 to develop practical recommendations for operational definitions of medication adherence, consistent with components of good research practice, as part of a broader initiative to improve measurement and analysis standards in adherence research. Key theoretical and methodological bases were the $\mathrm{ABC}$ adherence taxonomy, the EMERGE guidelines and the three-step measurement process well-established in psychometric literature. ${ }^{15}$ The first step of this measurement process - definition - identifies the attribute(s) to be measured and is represented in adherence research by the $\mathrm{ABC}$ taxonomy. The second step - operationalization - ascertains what needs to be done practically to know how the attribute(s) manifest(s) in a particular case; it thus translates the definition into a set of operations by which the previously defined attribute(s) will be observed in the study sample, and thus arrives at a more precise operational definition. The third step - quantification - translates operational definitions into numbers and thus allows data analysis and aggregation into summary measures. 
The group aimed to articulate general operationalization recommendations that would be consistent with the $\mathrm{ABC}$ and EMERGE principles and applicable to various types of medications, health conditions, healthcare systems, research designs, and data sources. By following this approach, researchers should be able to identify whether/how the $\mathrm{ABC}$ phases apply to their own study, and which measures are most appropriate for their needs. Group members used their experience in adherence research to assess critically the applicability of the principles proposed to various examples of adherence research.

The study was funded by ESPACOMP, and group members co-authored this article. We present below the resulting recommendations with examples of research applications and discuss their implications and further developments necessary.

\section{Results}

The working group identified four main sources of variation that impact decisions concerning the measurement of adherence in clinical trials and routine clinical care. We present them in order of importance relative to defining adherence as a temporal sequence. First, treatment timelines may vary substantially for the same medication regimen, depending on prescribing, dispensing, dosing and usage practices. While these timelines may be more tightly controlled in clinical trials, treatment regimens become less standardized in clinical practice as they are adapted to patients' individual needs and preferences. Second, key events occurring on these timelines, such as starting and stopping medication, may vary between participants in clinical trials despite strict protocols, and even more so in routine care, where changing or reinitiating treatment may occur, either in agreement with the treating physician or independently. Third, studies may have various objectives, such as studying adherence predictors or consequences, accounting for the influence of non-adherence on drug exposure and efficacy, improving individuals' adherence, or driving organization- or system-wide change. Depending on these objectives, the study design beyond adherence measurement may need adjustments depending on how related variables are placed in 
the temporal sequence described by adherence timelines and events. Fourth, various data sources may be available, with different limitations and possibilities, which may restrict how research questions are formulated and the type of evidence that can be generated about this temporal sequence.

Due to these sources of variation, no single measurement method of adherence is appropriate in all situations. Instead, careful consideration of these domains would lead to selecting the most appropriate measure for each study. We therefore introduce TEOS (Timelines - Events - Objectives - Sources, Figure 1), a four-component iterative process that researchers can follow to include these considerations in adherence measurement and record decisions transparently.

Figure 1: The TEOS framework

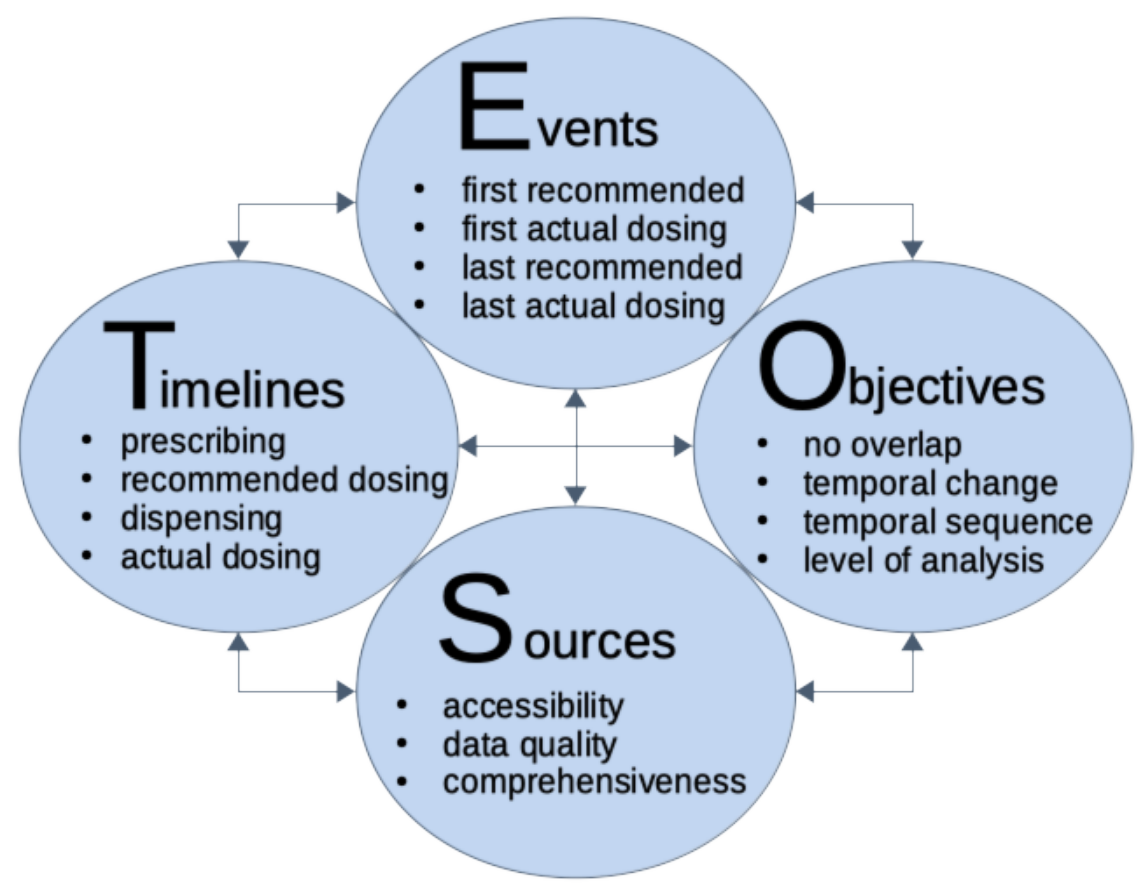

\section{The TEOS framework}

Timelines - what are the temporal characteristics of the medication regimen(s) under study?

Adherence to medications is defined broadly as the process by which patients take their medications as prescribed. This implies comparing two timelines: recommended and actual 
dosing events. As patients can only use medications if these are available to them, we also need to consider prescribing and dispensing events. Therefore, a first step is to understand conceptually how prescribed and actual use occurs in real life for each medication in the study context and represent this information explicitly on four parallel timelines:

1. Prescribing events: start dates and durations of prescriptions,

2. Recommended dosing events (dosing regimen): quantity (e.g. two tablets) and frequency (e.g. twice daily) of the recommended sequence of dosing events during prescription intervals,

3. Dispensing events: dates and durations of dispensations related to the specific prescriptions, which make medications available to patients, and

4. Actual dosing events: date and time of administration of available medications.

Figure 2 provides a representation of these timelines for one prescribed medication. They occur simultaneously during a period of interest (observation window; e.g. a year, or a month), before or after a specific event (index date) in a broader time period around the observation window on which data may be available (follow-up window). The index date may be a clinical event, a consultation, or a first prescription of a specific medication after a period of no treatment. Mapping these timelines on each patient's medication history is the first step towards accurate adherence measurement. 
Figure 2: Four timelines of medication adherence: Prescribed use with prescriptions and recommended dosing events and Actual use with dispensations and actual dosing events. Dashed rectangles: Follow-up window (black) and observation window (green).

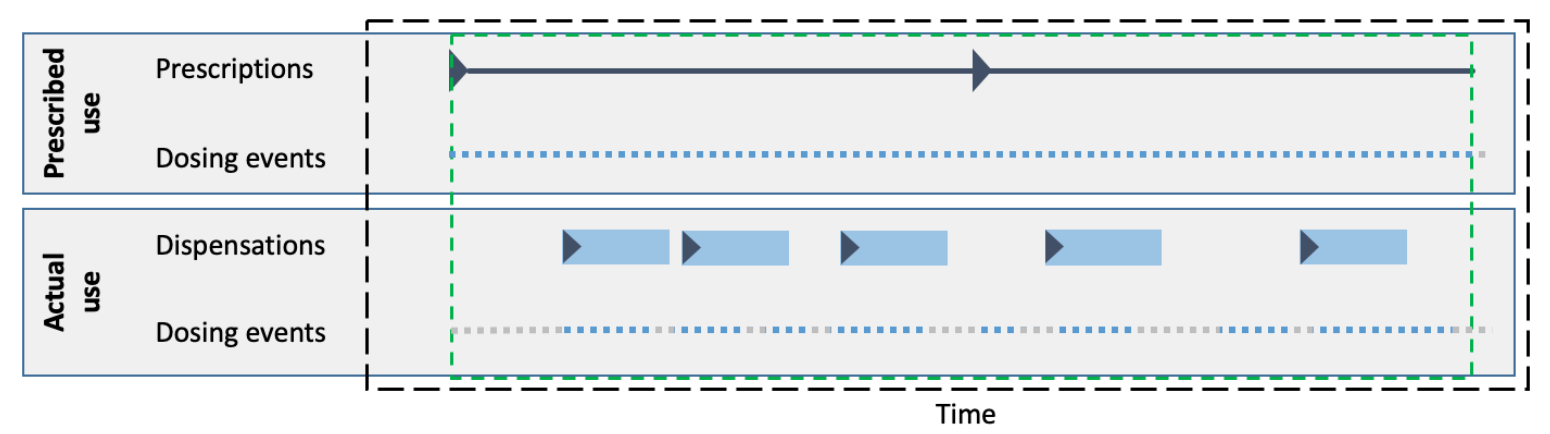

Key events - what are the boundaries delimiting adherence phases?

The ABC taxonomy delimits the three adherence phases by four key events: (1) first recommended dosing event, (2) first actual dosing event ("initiation"), (3) last actual dosing event (“discontinuation"), and (4) last recommended dosing event. These four events are boundaries for all other prescribed and actual dosing events. Between a first and a last actual dosing event, the time-series of actual dosing events may correspond to a variable extent to the time-series of recommended dosing events; this variability is captured by the concept of "implementation". In routine care, patients may discontinue (event 3) and reinitiate (event 2) treatments multiple times during a period covered by the same prescription. At the same time, prescriptions may be stopped by a provider (event 4) and then restarted (event 1) at a later date. Identifying these events represents the second step in measuring adherence, as it allows the investigator to distinguish between the three adherence phases and subsequently identify suitable adherence metrics for each phase. Depending on the follow-up window length, the timelines may include one or more of the four key events, e.g. the probability of discontinuation increases with the duration the follow-up window. Understanding how the timing of these events varies is essential for deciding the timing of observation windows for adherence calculations within the same study, as well as for explaining inconsistencies in results between studies. 
Additional events may occur within an implementation phase, such as changes in recommended dosage and generic or therapeutic substitution which need particular consideration when assessing adherence outside controlled environments. ${ }^{16}$ These situations may be followed by a recommendation to finish the previous supply with the same or the new dosage regimen, or to discard any remaining medication and start the new prescription. Dose changes may also occur between prescription events, for example when prescribed regimens include recommendations to step up or down dosage depending on clinical need. These events may impact measurement, and therefore need to be recorded and considered in sample selection or adherence calculations.

\section{Research objectives and design - what adherence-related evidence we want?}

Operationalization is strongly related to research design, and the choice of adherence measures can impact relationships between predictors and outcomes. ${ }^{17}$ Understanding in depth the temporal characteristics of treatments and how we can delimit adherence phases will generate deeper insights into the adherence-related evidence we need and can generate, with new and perhaps unanticipated implications for research objectives and study design. We therefore recommend that researchers reflect on how the adherence-related variables they aim to study (predictors, outcomes, mediators, moderators, potential confounders) are situated on the timelines described above. Without aiming to be comprehensive, we highlight here four examples of such considerations: 1) are these variables measured separately from adherence behaviors?; 2) do they change over time?, 3) what is their temporal sequencing in relation to adherence?, and 4) at what levels of analysis do they vary? For most studies, answering these questions would improve substantially the quality of the study design and the interpretation of results.

Operational definitions of adherence require clear boundaries between the actual behavior, as per the $\mathrm{ABC}$ taxonomy, and other proximal variables. Irrespective of the adherence measure selected, valid causal inference can be achieved only if the measure has little or no measurement overlap with how adherence determinants and outcomes are measured. For 
example, when examining relationships between adherence determinants and behaviors, researchers should avoid self-reported adherence scales that conflate questions on behaviors and determinants into single scores. Such scales are common in the literature. ${ }^{18}$

Once conceptual boundaries are established, an analysis of the temporal properties of these variables would inform the timing of adherence measurement in relation to the study hypotheses. These variables may be time-invariant or time-varying properties, or single events. Their duration and how much they change in time should be considered when choosing the timing, frequency and duration of measurement for adherence, and the relevant adherence phases. Identifying the temporal sequence between adherence phase(s) and adherence-related variables is necessary for causal claims. For example, adherence would need to be measured after predictors and before outcomes, and the time lag between measurements should reflect causal effects that are biologically plausible.

In thinking about adherence predictors and outcomes, it is important to note that healthcare systems are naturally hierarchical and causal influences can occur at multiple "levels." Patients' beliefs about health and healthcare, income, and access to health are examples of adherence predictors at the patient level which may remain stable during a study and thus amenable to investigation at between-person level. Other adherence predictors, such as symptom severity or emotional states, may vary and thus have causal effects within-person. Healthcare professionals who prescribe and supply medicines may support or undermine medication adherence in a variety of ways, such as their interest and skill in collaborating with patients on medication taking, or the organizational infrastructure they establish for prescription refills or patient support. Similarly, some predictors are stable and be amenable to study at between-person level, while others may vary and have causal effects within-person, i.e. healthcare professional. Finally, there may be city, county, regional, or national-level factors affecting care. Both study questions and methods should reflect this multilevel nature of healthcare delivery ${ }^{19}$ and aim to 
detect and explain relevant variation at within- or between-patient level, healthcare professional, organization, or policy levels. ${ }^{20}$ The choice of analysis level(s) will depend on the practical applications envisaged, e.g. adherence indicators at practice level are less useful for intervention at patient level, but could be a target of healthcare professional behavior change. ${ }^{21}$ It will result in different temporal dynamics to be captured, as some phenomena may have higher temporal variation within-patient and more stability at policy level. For example, if we aim to produce evidence for managing adherence in provider-patient consultations, we might focus on individuallevel implementation patterns or persistence measures and a short- to medium timeframe. Alternatively, if we are interested in organization-level or system-wide diagnosis and change, we might examine population-level initiation and persistence distributions. The limited awareness of the importance of levels of analysis in adherence research so far is detrimental to the field, as evidence produced at one level is often interpreted at another. ${ }^{22}$ We therefore encourage researchers to perform this mapping exercise explicitly and refine research objectives in light of these considerations.

\section{Data sources - what is accessible?}

In order to match what we want to know with what we can know in specific settings, operational definitions need to consider the possibilities and limitations of available data sources in relation to the evidence required, which in essence is the temporal sequence of actual versus prescribed dosing and its place within the broader causal process involving the treatment regimen. The most common data sources in adherence research are electronic healthcare databases (e.g. electronic health records, claims, record linkage systems), ${ }^{23}$ electronic monitoring devices, and self-reports. Advantages of different data sources and measures have been discussed elsewhere in relation to adherence stages. ${ }^{2,9,10}$

To maximize data quality, it is essential to evaluate several data sources potentially available for research with respect to quality dimensions specific to each source (e.g. reliability, 
validity, responsiveness, and interpretability for self-report). ${ }^{24}$ This evaluation needs to be performed in relation to the adherence phase(s) and research objectives targeted. A single data source may contain information about one or multiple timelines and therefore makes it possible to compute markers for one or more adherence phase(s). Examples include: a dated note in an electronic health record indicating that a prescription was written; a medication plan detailing the prescribed regimen; a date in an administrative dataset showing that a prescription was filled; a smart (electronic) package opening event with date and time; a self-reported statement of no missing dose in the previous week; and a dated note in a health record that a medication was represcribed after a 6-month gap. Alternatively, combining multiple data sources may reduce bias and measurement error by aggregating complementary information on different timelines and events to generate a comprehensive data set and check consistency, e.g. in composite measures ${ }^{25}$ or by linking prescribing and dispensing data. ${ }^{26}$

These four types of information - timelines, events, objectives, and sources - are necessary to describe the events required to measure adherence according to the $\mathrm{ABC}$ taxonomy, and the temporal dynamics of the relationships of interest. Sometimes all the desired information is directly available, but more often we can only approximate the theoretical time series of prescribed and actual dosing events. For example, we infer from a first dispensing event that someone started a treatment (initiation), from a smart package opening event that someone took a dose (implementation), or from a certain gap between refill events that medication taking has been discontinued (persistence).

\section{Using the framework}

We therefore propose to consider, when constructing operational definitions of adherence, a series of questions addressing the four TEOS components (Table 1). The question order in Table 1 highlights the importance of adherence timelines as described above, but the process we propose is by no means linear. As TEOS components interact, the order in which the 
questions are tackled in a specific study may vary and decisions in one component may require adjustments in another. For example, decisions related to data sources following quality evaluation may induce changes to study design features, such as inclusion criteria and measurement of other variables. Answering these questions might prove straightforward in some contexts, for example if the study population is homogeneous and information is easily available, while in others more research might be necessary to support operationalization choices, such as method validation or sensitivity analyses. Nevertheless, we believe that these questions would help researchers make informed choices on operational definitions of adherence.

Table 1: Examples of questions to consider for each TEOS dimension.

\begin{tabular}{|l|l|}
\hline TEOS & $\begin{array}{l}\text { Examples of questions to consider for each prescribed/recommended } \\
\text { Dedicine in the research setting }\end{array}$ \\
\hline Timelines & $\begin{array}{l}\text { Prescribing events: } \\
\text { 1. When are the prescriptions issued? } \\
\text { 2. How long are they valid for? } \\
\text { 3. When are they renewed? } \\
\text { Recommended dosing events: } \\
\text { 1. When is dosing expected to begin after prescription? } \\
\text { 2. Are doses and dosing intervals (frequency of administration) fixed or do } \\
\text { they vary within patients, between patients or at other levels? If they } \\
\text { vary, what is the range of values? } \\
\text { 3. Do medication effects depend on additional recommendations? (e.g. inhaler } \\
\text { technique, administer with food, etc.) } \\
\text { Dispensing events: } \\
\text { 1. For how many days are medications supplied? } \\
\text { 2. What is the expected refill frequency? } \\
\text { 3. Does automatic dispensing occur? } \\
\text { Actual dosing events: } \\
\text { 1. What are the administration routes? } \\
\text { 2. How can patients modify medications (e.g. tablet splitting)? } \\
\text { 3. If additional recommendations apply (e.g. with food), are these followed } \\
\text { when administering? }\end{array}$ \\
$\begin{array}{l}\text { General questions in relation to all events: } \\
\text { 1. When does the follow-up window (time for which data are available) start } \\
\text { and end? }\end{array}$ \\
\hline
\end{tabular}




\begin{tabular}{|c|c|}
\hline & $\begin{array}{l}\text { 2. When does the observation window (time for which adherence is measured) } \\
\text { start and end? } \\
\text { Specific questions about particular events } \\
\text { 1. First recommended dosing event: What minimum duration without } \\
\text { prescribed use should precede a recommended dosing event indicating } \\
\text { the start of a new treatment? } \\
\text { 2. First actual dosing event: What is the maximum duration between first } \\
\text { recommended dosing event and first actual dosing event before non- } \\
\text { initiation is assumed? } \\
\text { 3. Last actual dosing event: What is the minimum duration with no dosing } \\
\text { events to differentiate between poor implementation and non- } \\
\text { persistence? } \\
\text { 4. Last recommended dosing event: } \\
\text { 1. How is the date of treatment end identified? } \\
2 \text { How are treatment changes/replacements differentiated } \\
\text { from treatment interruptions? }\end{array}$ \\
\hline Objectives & $\begin{array}{l}\text { Intervention: What intervention(s) will be provided with a potential impact on } \\
\text { medication adherence? } \\
\text { Predictors: What potential predictors of adherence will be measured and/or } \\
\text { targeted by the intervention(s)? } \\
\text { Outcomes: What outcomes will be measured? How are they affected by } \\
\text { medication adherence? } \\
\text { Moderators/mediators: What variables will be measured? How do they } \\
\text { intervene in the causal process? } \\
\text { General questions in relation to all variables: } \\
\text { 1. Are they measured separately from adherence behaviors? } \\
\text { 2. Do they change in time (i.e., time-varying)? } \\
\text { 3. What is the temporal sequencing in relation to adherence? } \\
\text { 4. At what levels of analysis do they vary? (within- or between-patients, } \\
\text { healthcare professionals, etc.) }\end{array}$ \\
\hline Sources & $\begin{array}{l}\text { 1. What data sources are available to derive the four } 4 \text { key events? } \\
\text { 2. What additional data are available to measure adherence? } \\
\text { 3. For what time period(s) are data available? } \\
\text { 4. How complete are the available datasets? } \\
\text { 5. What is the validity/reliability of the available data? } \\
\text { 6. What are the options for additional data collection? } \\
\text { 7. What are potential sources of measurement error? }\end{array}$ \\
\hline
\end{tabular}

After this exercise, operational definitions can be formulated for all relevant adherence measures consistent with the ABC taxonomy. We provide an example for reporting the TEOS exercise in tabular form (Table 2) and as graphical representation (Figure 2) for an intervention 
study to support medication adherence for patients starting any new chronic treatment. The operational definitions for each adherence phase are provided in Box 1, together with possible quantification and summary measures. Two additional examples are presented in Appendix 1.

Table 2: TEOS for an intervention study to support medication adherence for patients starting any new chronic treatment. The intervention will be provided in cluster-randomized pharmacies within 6 weeks after the first dispensing event and consist of 2 interviews, the first until up to 3 weeks, and the last at 4-6 weeks after the start of the treatment. We want to measure the impact of the intervention on short-term adherence (example adapted from published study). ${ }^{34}$

\begin{tabular}{|c|c|}
\hline Timelines: & $\begin{array}{l}\text { Prescription events: one first prescription issued, typically for } 30 \text { days } \\
\left.\text { (duration } \mathrm{D}_{1}\right) \text {, that can be filled } 1 \text { time and is followed by subsequent } \\
\left.\text { prescriptions for long-term use ( } 6+\text { months, } \mathrm{D}_{2}\right) \\
\text { Recommended dosing events: treatments will be prescribed to start } \\
\text { immediately, for regular use (e.g. daily to weekly, dosing varies; 'as needed' } \\
\text { use is excluded), } \\
\text { Dispensing events: the first dispensing event can happen within } 30 \text { days after } \\
\text { the first prescription and covers up to } 30 \text { days }\left(\mathrm{D}_{3}\right) \text {. Subsequent dispensing } \\
\text { events may cover up to } 90 \text { days }\left(\mathrm{D}_{4}\right) \text {. Automatic dispensing does not occur. } \\
\text { Actual dosing events: patients are expected to self-administer their } \\
\text { medication, the mode of administration varies, any type of modification is } \\
\text { possible (e.g. reducing/increasing doses, skipping/delaying administration; } \\
\text { seasonal variability may play a role for some medications and needs to be } \\
\text { considered when selecting medications for the study). }\end{array}$ \\
\hline Events: & $\begin{array}{l}\left.\text { First recommended dosing event: date of first prescription (time } \mathrm{T}_{0}\right) \text { after a } \\
\text { 1-year baseline period with no prescriptions for the same substance - it } \\
\text { represents the start of the observation window }\left(\mathrm{OW}, \mathrm{T}_{0}\right) \\
\text { First actual dosing event: occurs after first dispensation }\left(\mathrm{T}_{1}\right) \text {, date may vary } \\
\text { but most participants are likely to initiate before the first interview which takes } \\
\text { place up to } 3 \text { weeks after first dispensation. } \\
\text { Last actual dosing event: possibly occurring anytime during the OW if } \\
\text { initiated, or after the end of the OW }\left(\mathrm{T}_{3}\right) \\
\text { Last recommended dosing event: date of prescription discontinuation (if } \\
\text { within the } \mathrm{OW}) \text {, or after the end of the } \mathrm{OW}\left(\mathrm{T}_{2}\right)\end{array}$ \\
\hline Objectives: & $\begin{array}{l}\text { The intervention will be initiated at the first dispensing event in the } \\
\text { pharmacy. We are interested in the short-term effects of this intervention on } \\
\text { patients' adherence, compared to a control group receiving usual care. We } \\
\text { want to measure the impact of the intervention on initiation, implementation } \\
\text { and persistence in the first } 10 \text { weeks after the first dispensing event. } \\
\text { We also aim to investigate the correlation between occurrence of side effects } \\
\text { and adherence at } 10 \text { weeks. }\end{array}$ \\
\hline
\end{tabular}




\begin{tabular}{|l|l|}
\hline & $\begin{array}{l}\text { Cluster randomization will occur at pharmacy level. Several patient-level } \\
\text { time-invariant (non-modifiable) characteristics will be controlled for. Health } \\
\text { system level variables are likely to remain constant. }\end{array}$ \\
\hline Sources: & $\begin{array}{l}\text { We can collect participants' self-reports of behavior and side effect } \\
\text { occurrence (standardized battery of items during the intervention). We also } \\
\text { have access to pharmacy refill dates and quantities (electronic records of } \\
\text { actual dispensing events) and prescription dates (manually entered by } \\
\text { pharmacy staff), but only for medications dispensed in the pharmacy } \\
\text { providing the first dispensation. Recommended dosages are not easily } \\
\text { accessible in routine data but could be obtained from patients or healthcare } \\
\text { professionals. Electronic monitoring is not feasible due to logistics, mainly } \\
\text { because of variations in mode of administration (oral, injection, spray, etc.). }\end{array}$ \\
\hline
\end{tabular}

Figure 3: Example for a graphical representation of the TEOS exercise. $D_{1}-D_{4}$ and $T_{0}-T_{3}$ refer to the timelines and events, respectively, mentioned in Table 2 above. Dashed rectangles: Follow-up window (black) and observation window (green). The intervention (orange line) is provided by healthcare professionals to patients and consists of two interviews which occur at up until 3 weeks and 4-6 weeks after intervention start; self-reported adherence data are collected at these times (orange diamonds). Additional predictors and outcomes are measured at the patient level (grey lozenge).

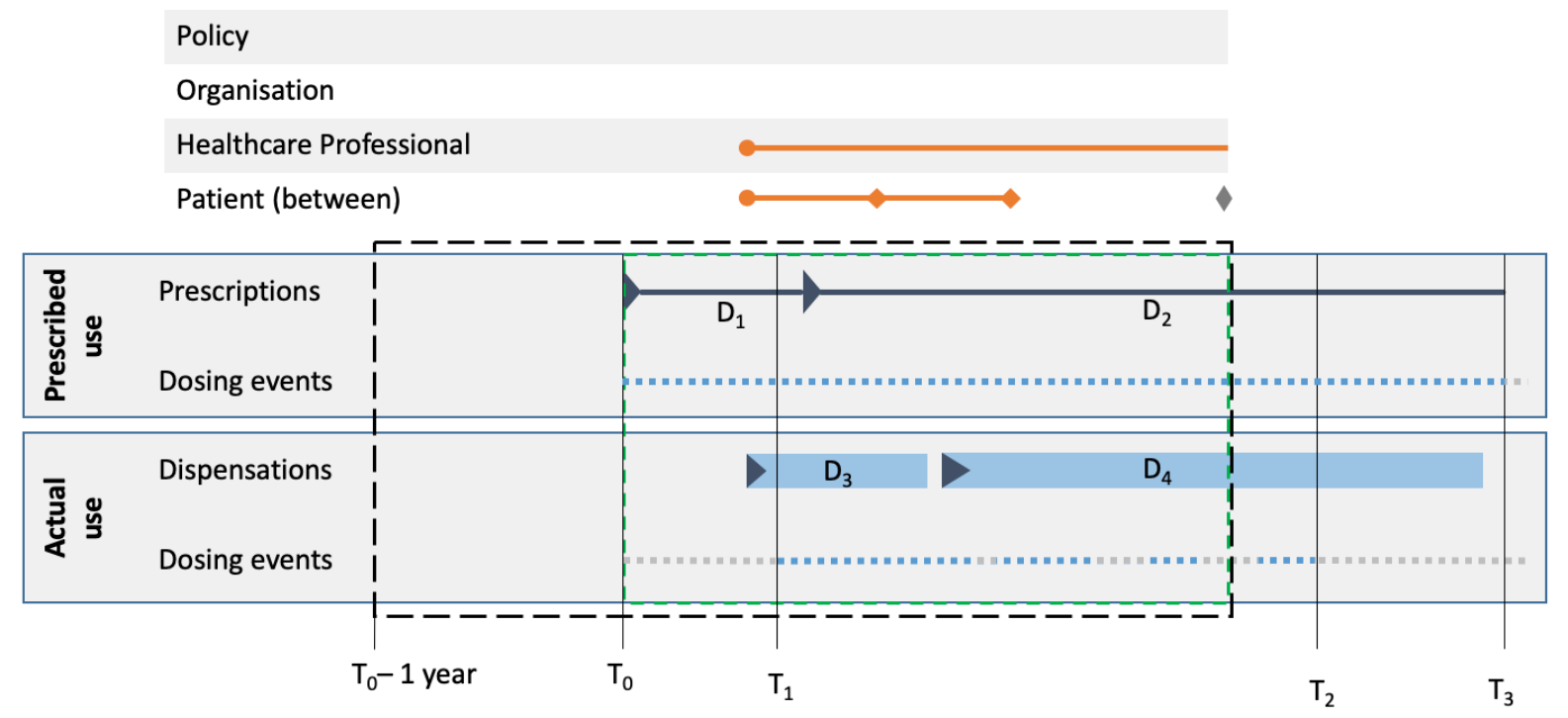

Box 1: Operational definitions for measuring adherence in the example study

\section{Initiation:}


Patients with at least one refill recorded within 10 weeks after the first dispensing event and who self-reported at least one actual dosing event within 10 weeks (data extracted from patient record).

Quantification: Binary variable (yes/no).

Summary measure: percentage initiating patients

\section{Persistence:}

Patients who initiated treatment, have no documented end of recommended use and selfreported at least one actual dosing event in week 10 (data extracted from patient record).

Quantification: Binary variable (yes/no).

Summary measure: percentage persistent patients

Implementation: Self-reported quality of implementation by patients who were persistent at week 10, as measured by the Three-Item Self-Report Measure for Medication Adherence at week $10^{35}$.

Quantification: Continuous variable as per scoring instructions (percentage, range $0-100$ ).

Summary measure: median implementation score (inter-quartile range)

\section{Discussion and conclusions}

A solid evidence base for adherence to medications requires shared standards and definitions, for which the ABC taxonomy and EMERGE guidelines have set the foundation. Applying them in practice requires shared procedures for understanding and measuring adherence processes in various clinical settings ${ }^{27}$. We propose TEOS, a four-component framework that researchers may use to perform a conceptual analysis of adherence timelines and key events in relation to research objectives and data sources. By using TEOS, we hope to encourage researchers to explore more options for study design and identify more accurate ways to measure adherence, formulate hypotheses and analyze data. Although full replication and cross-study comparability will always be difficult to achieve as rarely two studies will share the same specifics, we believe that reporting operational definitions using TEOS will improve transparency and reproducibility of adherence studies and thus facilitate comparisons.

TEOS highlights the fact that many important research questions regarding medication adherence are not being asked with sufficient rigor. Prior reviews have noted that initiation and persistence are less studied than implementation, ${ }^{1}$ and adherence has been studied more at patient level than at the provider or health care site levels. ${ }^{20}$ Moreover, TEOS points to several 
key limitations in previous studies regarding the temporal properties of adherence and its multilevel variation. ${ }^{19}$ Not separating adherence phases is common, and a source of measurement error and confusion in interpreting results. ${ }^{28}$ Current interventions targeting changes in adherence within-person rely mostly on evidence at the between-person level, which may be subject to different influences; distinguishing between- and within-person variation in adherence holds promise for improving adherence interventions. ${ }^{22}$ We hope that the explicit use of TEOS will stimulate new research in these less explored areas, and result in the formulation of more precise hypotheses which can generate more actionable evidence for adherence management.

The TEOS framework focuses on operationalization and design of research concerning medication adherence and is intended to stimulate debate and promote consensus among the research community. The format of the examples we give of operational definitions represents an initial proposal for discussion. We intentionally did not review or endorse specific adherence measures or approaches to quantification or aggregation, as their appropriateness depends on the interactions between the four components we illustrated here and the relevance of the different choices to specific settings. Several options for summarizing adherence across values (e.g. median implementation percentage) or across patients (e.g. percentage of patients implementing as prescribed over a specific threshold value) have been proposed and raise various analysis challenges ${ }^{29}$ which are beyond the scope of this article.

TEOS is described here for a single medication, yet long-term treatments typically involve multiple medications (polypharmacy), often aiming for non-additive effects. ${ }^{30,31}$ Adherence timelines may differ substantially between medications, resulting in potential overlapping intervals of initiation, persistence/implementation, and non-persistence for different medications. In certain cases, it might be useful to consider adherence to a treatment consisting of multiple medications instead of single medications. ${ }^{32,33}$ Treatment effects - and consequently, adherence 
- of some medications are importantly affected by the concomitant/sequential use of other medications, or other substances, such as food, etc.; these other events may represent additional layers of information to consider in adherence studies. Operational definitions of polypharmacy could build on the TEOS framework and add questions specific to multiple medication regimens.

The ESPACOMP adherence methodology working group aims to provide the research community with practical tools for developing a high-quality evidence base for adherence management decisions from individual to policy level. TEOS is part of this objective, as well as of the broader movement towards transparency and reproducibility in research. Its application to different medication regimens and clinical settings would advance our understanding of adherence to medications and the role of adherence management in improving population health.

Funding support: This work was supported by the International Society for Medication Adherence (ESPACOMP). AD was supported by the European Commission (European Community's 7th Framework GA282593 and H2020 programme MSCA-IF GA706028) and IDEXLyon (ANR-16-IDEX- 0005); SA was supported by the Swiss Science Foundation (P2BSP3_178648). DH receives support from Health and Care Research Wales (Senior Research Leader, SRL-19-18) and the MRC-NIHR (Trials Methodology Research Partnership, MR/S014357/1). IW is partially supported by the Providence/Boston Center for AIDS Research (P30AI042853) and by the National Institute of General Medical Sciences of the National Institutes of Health, which funds Advance Clinical and Translational Research (Advance-CTR) from the Rhode Island Institutional Development Award IDeA-CTR award (U54GM115677).

Acknowledgements: The authors would like to thank Dr. Remon Helmy for his participation in group discussions on the adherence operationalization framework and reviewing manuscript versions. 


\section{References}

1. Dima AL, Hernandez G, Cunillera O, Ferrer M, de Bruin M, the ASTRO-LAB group. Asthma inhaler adherence determinants in adults: systematic review of observational data. Eur Respir J. 2015;45(4):994-1018. doi:10.1183/09031936.00172114

2. Whalley Buono E, Vrijens B, Bosworth HB, Liu LZ, Zullig LL, Granger BB. Coming full circle in the measurement of medication adherence: opportunities and implications for health care. Patient Prefer Adherence. 2017;11:1009-1017. doi:10.2147/PPA.S127131

3. Jönsson AK, Schiöler L, Lesén E, Sundell KA, Mårdby A-C. Influence of refill adherence method when comparing level of adherence for different dosing regimens. Eur J Clin Pharmacol. 2014;70(5):589-597. doi:10.1007/s00228-014-1649-2

4. Orrell C, Cohen K, Leisegang R, Bangsberg DR, Wood R, Maartens G. Comparison of six methods to estimate adherence in an ART-naive cohort in a resource-poor setting: which best predicts virological and resistance outcomes? AIDS Res Ther. 2017;14(1):20. doi:10.1186/s12981-017-0138-y

5. Souverein PC, Koster ES, Colice G, et al. Inhaled Corticosteroid Adherence Patterns in a Longitudinal Asthma Cohort. J Allergy Clin Immunol Pract. 2017;5(2):448-456.e2. doi:10.1016/j.jaip.2016.09.022

6. El Alili M, Vrijens B, Demonceau J, Evers SM, Hiligsmann M. A scoping review of studies comparing the medication event monitoring system (MEMS) with alternative methods for measuring medication adherence. Br J Clin Pharmacol. 2016;82(1):268-279. doi:10.1111/bcp.12942

7. Garber M, Nau D, Erickson S, Aikens J, Lawrence J. The Concordance of Self-Report With Other Measures of Medication Adherence: A Summary of the Literature. [References]. Med Care. 2004;42(7):649-652. doi:http://dx.doi.org/10.1097/01.mlr.0000129496.05898.02

8. Vrijens $B$, De Geest $S$, Hughes DA, et al. A new taxonomy for describing and defining adherence to medications. Br J Clin Pharmacol. 2012;73(5):691-705. doi:10.1111/j.13652125.2012.04167.x

9. Elseviers $M, V$ Vrijens $B$. Assessment of medication adherence in field research. In: Elseviers M, Wettermark B, Almarsdóttir AB, et al., eds. Drug Utilization Research. Chichester, UK: John Wiley \& Sons, Ltd; 2016:361-368. doi:10.1002/9781118949740.ch35

10. Lehmann A, Aslani $\mathrm{P}$, Ahmed $\mathrm{R}$, et al. Assessing medication adherence: options to consider. Int J Clin Pharm. 2013;36(1):55-69. doi:10.1007/s11096-013-9865-x

11. Williams AB, Amico KR, Bova C, Womack JA. A Proposal for Quality Standards for Measuring Medication Adherence in Research. AIDS Behav. 2012;17(1):284-297. doi:10.1007/s10461-012-0172-7 
12. Vollmer WM, Xu M, Feldstein A, Smith D, Waterbury A, Rand C. Comparison of pharmacybased measures of medication adherence. BMC Health Serv Res. 2012;12(1):155. doi:10.1186/1472-6963-12-155

13. Zarin DA, Tse T, Williams RJ, Califf RM, Ide NC. The ClinicalTrials.gov Results Database Update and Key Issues. N Engl J Med. 2011;364(9):852-860. doi:10.1056/NEJMsa1012065

14. De Geest S, Zullig LL, Dunbar-Jacob J, et al. ESPACOMP Medication Adherence Reporting Guideline (EMERGE). Ann Intern Med. 2018;169(1):30-35. doi:10.7326/M18-0543

15. Thorndike RM, Thorndike-Christ TM. Measurement and Evaluation in Psychology and Education. 8 edition. Boston: Pearson; 2009.

16. Arnet I, Kooij MJ, Messerli M, Hersberger KE, Heerdink ER, Bouvy M. Proposal of Standardization to Assess Adherence With Medication Records: Methodology Matters. Ann Pharmacother. 2016;50(5):360-368. doi:10.1177/1060028016634106

17. Dunbar-Jacob J, Rohay JM. Predictors of medication adherence: fact or artifact. J Behav Med. 2016;39(6):957-968. doi:10.1007/s10865-016-9752-8

18. Nguyen T-M-U, Caze AL, Cottrell N. What are validated self-report adherence scales really measuring?: a systematic review. Br J Clin Pharmacol. 2014;77(3):427-445. doi:10.1111/bcp.12194

19. Meyers DJ, Cole MB, Rahman $\mathrm{M}$, et al. The association of provider and practice factors with HIV antiretroviral therapy adherence. AIDS. 2019;33(13):2081-2089. doi:10.1097/QAD.0000000000002316

20. Berben L, Dobbels F, Engberg S, Hill MN, De Geest S. An Ecological Perspective on Medication Adherence. West J Nurs Res. 2012;34(5):635-653. doi:10.1177/0193945911434518

21. Genberg $\mathrm{BL}$, Rogers $\mathrm{WH}$, Lee $\mathrm{Y}$, et al. Prescriber and pharmacy variation in patient adherence to five medication classes measured using implementation during persistent episodes. Pharmacoepidemiol Drug Saf. 2016;25(7):790-797. doi:10.1002/pds.4025

22. Dima AL, van Ganse $E$, Stadler G, de Bruin M. Does adherence to inhaled corticosteroids predict asthma-related outcomes over time? A cohort study. Eur Respir J. 2019; 54:1900901. doi:10.1183/13993003.00901-2019

23. Pacurariu A, Plueschke K, McGettigan P, et al. Electronic healthcare databases in Europe: descriptive analysis of characteristics and potential for use in medicines regulation. $B M J$ Open. 2018;8(9):e023090. doi:10.1136/bmjopen-2018-023090

24. Mokkink LB, Terwee CB, Patrick DL, et al. The COSMIN checklist for assessing the methodological quality of studies on measurement properties of health status measurement instruments: an international Delphi study. Qual Life Res. 2010;19(4):539549. doi:10.1007/s11136-010-9606-8 
25. Rotzinger A, Cavassini M, Bugnon O, Schneider MP. Development of an algorithm for analysing the electronic measurement of medication adherence in routine HIV care. Int J Clin Pharm. 2016;38(5):1210-1218. doi:10.1007/s11096-016-0354-x

26. García-Sempere A, Hurtado I, Sanfélix-Genovés J, Rodríguez-Bernal C, Peiró S, SanfélixGimeno G. Improving the accuracy of medication adherence measures using linked prescription and dispensation data: findings from the ESOSVAL cohort of patients treated with osteoporosis drugs. Curr Med Res Opin. 2019;0(0):1-10. doi:10.1080/03007995.2019.1601944

27. Khan MU, Kohn M, Aslani P. The need for a paradigm shift in adherence research: The case of ADHD. Res Soc Adm Pharm. 2019;15(3):318-320. doi:10.1016/j.sapharm.2018.04.033

28. Vrijens B, Vincze G, Kristanto P, Urquhart J, Burnier M. Adherence to prescribed antihypertensive drug treatments: longitudinal study of electronically compiled dosing histories. BMJ. 2008;336(7653):1114-1117. doi:10.1136/bmj.39553.670231.25

29. Dunbar-Jacob J, Houze MP, Kramer C, Luyster F, McCall M. Adherence to Medical Advice: Processes and Measurement. In: Steptoe A, ed. Handbook of Behavioral Medicine: Methods and Applications. New York, NY: Springer; 2010:83-95. doi:10.1007/978-0-38709488-5_7

30. Choudhry NK, Shrank WH, Levin RL, et al. Measuring Concurrent Adherence to Multiple Related Medications. Am J Manag Care. 2009;15(7):457-464.

31. Martin BC, Wiley-Exley EK, Richards S, Domino ME, Carey TS, Sleath BL. Contrasting Measures of Adherence with Simple Drug Use, Medication Switching, and Therapeutic Duplication. Ann Pharmacother. 2009;43(1):36-44. doi:10.1345/aph.1K671

32. Youn B, Shireman TI, Lee $Y$, Galárraga O, Wilson IB. Trends in medication adherence in HIV patients in the US, 2001 to 2012: an observational cohort study. I Int AIDS Soc. 2019;22(8):e25382. doi:10.1002/jia2.25382

33. Youn $\mathrm{B}$, Shireman $\mathrm{Tl}$, Lee $\mathrm{Y}$, et al. Ten-year trends in antiretroviral therapy persistence among US Medicaid beneficiaries. AIDS. 2017;31(12):1697-1707. doi:10.1097/QAD.0000000000001541

34. Elliott RA, Boyd MJ, Salema N-E, et al. Supporting adherence for people starting a new medication for a long-term condition through community pharmacies: a pragmatic randomised controlled trial of the New Medicine Service. BMJ Qual Saf. 2016;25(10):747-758. doi:10.1136/bmjqs-2015-004400

35. Wilson IB, Lee Y, Michaud J, Fowler FJ, Rogers WH. Validation of a New Three-Item SelfReport Measure for Medication Adherence. AIDS Behav. 2016;20(11):2700-2708. doi:10.1007/s10461-016-1406-x 


\section{Appendix 1}

Table 1: TEOS for an observational study on adherence to an ongoing chronic treatment (more than 6 months covered by prescriptions in 12 months before inclusion in the study). We want to examine the variation in adherence to inhaled asthma medication within and between individuals, and between healthcare professionals; and its determinants and effects on asthma control (example adapted from published study). ${ }^{1}$

\begin{tabular}{|c|c|}
\hline Timelines: & $\begin{array}{l}\text { Prescription events: prescriptions issued usually for } 6 \text { months }\left(\mathrm{D}_{1}\right) \text { and } \\
\text { renewed during follow-up consultations }\left(\mathrm{D}_{2} \ldots\right) \text {; prescription duration may } \\
\text { vary; treatment should continue without gap once prescribed unless } \\
\text { prescription is specifically discontinued by the prescriber. Yet, actual gaps } \\
\text { between prescriptions may occur (e.g. due to missing/late follow-up } \\
\text { meetings); if prescription is discontinued, it is possible that a new prescription } \\
\text { occurs within the observation window (OW) or after. } \\
\text { Recommended dosing events: treatments should start immediately after the } \\
\text { first prescription, for daily use; dosing recommendations may change during } \\
\text { the course of the treatment for an individual, and vary between individual } \\
\text { patients, and between prescribers ('as needed' use is excluded). } \\
\text { Dispensing events: dispensing events usually start within } 30 \text { days of the first } \\
\left.\text { prescription date and cover between } 30 \text { and } 90 \text { days ( } \mathrm{D}_{3}, \mathrm{D}_{4} . . .\right) \text {; automatic } \\
\text { dispensing does not occur. } \\
\text { Actual dosing events: the medication is administered by patients themselves } \\
\text { or caregivers (e.g. parents), medication is administered by inhalation, } \\
\text { effectiveness depends on the quality of inhalation (inhaler technique); patients } \\
\text { can administer less or more inhalations, skip an administration, take earlier or } \\
\text { later during the day, or administer incorrectly. }\end{array}$ \\
\hline Events: & $\begin{array}{l}\text { Study participation may last for up to } 2 \text { years; routine care data can be } \\
\text { available for a follow-up window starting } 1 \text { year before inclusion, and possibly } \\
\text { for several months after the end of study participation. } \\
\text { First recommended dosing event: date of first prescription }\left(\mathrm{T}_{0}\right) \text { after at least } \\
6 \text { months with no prescriptions for the same substance - occurs before the OW } \\
\text { but may reoccur during the OW if preceded by a prescription end. } \\
\text { First actual dosing event: occurs after first dispensation }\left(\mathrm{T}_{1}\right) \text {, which occurs } \\
\text { before the OW or anytime during the OW if preceded by prescription end. } \\
\text { Last actual dosing event: assumed based on estimated end of dispensed } \\
\text { supply given dosing recommendations, or accessed directly by self-report or } \\
\text { electronic monitoring - may occur anytime during the OW or after the end of } \\
\left.\text { the OW (T } \mathrm{T}_{2}\right) \\
\text { Last recommended dosing event: date of prescription discontinuation } \\
\text { (prescription end followed by at least } 6 \text { months with no prescriptions for the } \\
\text { same substance) - occurs within the OW or after the OW }\left(\mathrm{T}_{3}\right)\end{array}$ \\
\hline
\end{tabular}




\begin{tabular}{|l|l|}
\hline Objectives: & $\begin{array}{l}\text { We are interested in within- and between person variation in inhaler adherence } \\
\text { in conditions of routine care, its predictors (determinants) and its effects on } \\
\text { asthma control. Adherence determinants and asthma control are measured } \\
\text { separately from adherence behaviors and represent distinct constructs. Asthma } \\
\text { control varies in time under the influence of multiple factors. Lowering } \\
\text { adherence or interrupting treatment may have a negative impact on asthma } \\
\text { control within several weeks. To our knowledge, there is no evidence } \\
\text { regarding temporal variation of determinants to inhaler adherence within- } \\
\text { patients and the lag of their effects on behaviors. Examples of adherence } \\
\text { determinants of interest are individual (e.g. illness and medication beliefs, } \\
\text { self-efficacy), interpersonal (e.g. trust in healthcare professional, social } \\
\text { support), related to healthcare provision (e.g. type of treatment, self- } \\
\text { management support delivered), and organization (e.g. location and size of } \\
\text { primary care practice). Both asthma control and adherence determinants can } \\
\text { impact adherence and be impacted by it; therefore, obtaining repeated } \\
\text { measures would allow examining bi-directional relationships. Both may vary } \\
\text { at different levels, e.g. within- and between-patients, healthcare } \\
\text { professionals, and organisation levels. No intervention is performed in this } \\
\text { study, except the usual care which may vary at HCP and organisation levels } \\
\text { and thus influence variation in adherence and asthma control at these levels. }\end{array}$ \\
$\begin{array}{l}\text { Patients (or their caregivers) can self-report behavior and asthma control via } \\
\text { telephone interviews with trained interviewers; to balance participant burden, } \\
\text { data collection costs and data granularity, interviews every } 4 \text { months are } \\
\text { considered feasible. Prescription data (dates, dosing recommendations, } \\
\text { duration) are manually entered by the General Practitioners recruiting } \\
\text { participants in the study. Dispensing data (dates, quantities, type of } \\
\text { medication) are available via electronic reimbursement information from the } \\
\text { national public healthcare system. Electronic monitoring is not feasible due } \\
\text { to cost, length of study participation (participant burden) and geographical } \\
\text { spread of the sample (national study). }\end{array}$ \\
\hline Sources:
\end{tabular}


Box 1: Operational definitions for measuring adherence in the example study

Initiation: Not studied (treatment interruption and re-initiation may occur occasionally, but are unlikely)

Persistence: Patients with no treatment gaps over 6 months during the study period (OW) based on supply dispensed during OW and 1 year before?; Alternative: Patients (or caregivers) declaring no discontinuation of treatment longer than 6 months during the study period, based on self-reported medication prescription and intake using Medication Intake Survey-Asthma ${ }^{2}$ Quantification: Binary variable (yes/no).

Summary measure: percentage persistent patients

Implementation: Self-reported quality of implementation (1-month taking adherence) as reported by patients (or caregivers) every 4 months (up to 7 values per participant) using the Medication Intake Survey-Asthma ${ }^{2}$.

Quantification: Continuous variable as per scoring instructions (percentage, range $0-100$ ), centered on participant averages for multi-level analysis.

Summary measure: median implementation score (inter-quartile range)

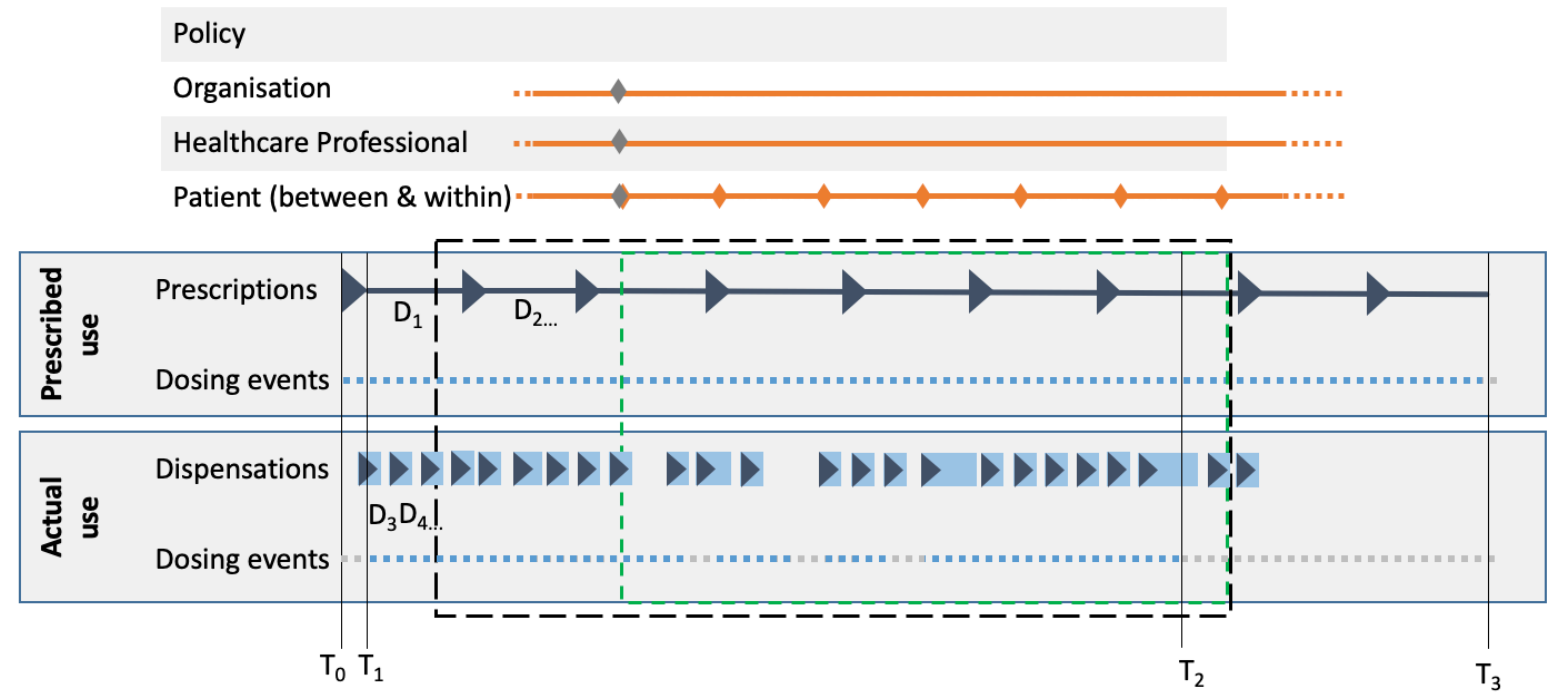

Figure 1: Example for a graphical representation of the TEOS exercise. $D_{1}-D_{4}$ and $T_{0}-T_{3}$ refer to the timelines and events, respectively, mentioned in Table 1 above. Dashed rectangles: Follow-up window (black) and observation window (green). Usual care (orange line) is provided by healthcare professionals to patients within different organisations. Adherence is measured at within patient level (orange lozenges) every 4 months for up to 2 years. Additional predictors and outcomes are measured at patient inclusion at between-patient, healthcare professional and organisation level (grey lozenges). 
Table 2: TEOS for an intervention study to support adherence to post-transplantation medication. The intervention is provided by nurses to patients $>1$ year post-transplant after a 3-month run-in period, and consists of 4 visits within 6 months, and a 6-month adherence follow-up period. We want to measure the impact of the intervention on post-intervention adherence (example adapted from published study). ${ }^{3}$

\begin{tabular}{|c|c|}
\hline Timelines: & $\begin{array}{l}\text { Prescription events: a first prescription was issued during hospital stay post } \\
\text { transplantation ( }>1 \text { year before study enrollment) and covered } 1-2 \text { months } \\
\left(\mathrm{D}_{1}\right) \text {; it was followed by subsequent prescriptions for } 2-4 \text { months }\left(\mathrm{D}_{2} \ldots\right) \\
\text { Recommended dosing events: treatment was prescribed to start immediately, } \\
\text { and medication dose adaptation may occur within the first year after } \\
\text { transplant; at study enrolment, only patients on twice daily dosing regimen are } \\
\text { included (to standardize adherence monitoring), i.e. one tablet every } 12 \pm 2 \\
\text { hours } \\
\text { Dispensing events: the first dispensing event occurred immediately after the } \\
\text { first prescription under strict supervision during hospital stay ( } \mathrm{D}_{3} ;>1 \text { year } \\
\text { before study enrollment), as part of induction therapy. At hospital discharge, } \\
\text { patients are followed up every two weeks to monitor adaptation to the new } \\
\text { treatment regimen, in concertation with the hospital or local pharmacy. } \\
\text { Patients may refill their prescriptions subsequently at their chosen pharmacy } \\
\left.\text { for } 1-4 \text { months ( } \mathrm{D}_{4} \ldots . .\right) \text {. Automatic dispensing does not occur. } \\
\text { Actual dosing events: patients self-administer their medication, mode of } \\
\text { administration is oral (tablets), patients may miss one or more consecutive } \\
\text { doses or may administer earlier or later. }\end{array}$ \\
\hline $\mathrm{E}$ & $\begin{array}{l}\text { First recommended dosing event: date of first prescription }\left(\mathrm{T}_{0}\right) \text { post- } \\
\text { transplant may be } \geq 1 \text { year before enrollment in the study (the start of the } \\
\text { follow-up window; FUW, } T_{0} \text { ) } \\
\text { First actual dosing event: occurs after first dispensation }\left(\mathrm{T}_{1}\right) \text {, all participants } \\
\text { have initiated before the start of the FUW }\left(\mathrm{T}_{0}\right) \text {. } \\
\text { Last actual dosing event: a small minority stop; such events are life- } \\
\text { threatening, unless the patient receives specialized care within a short period } \\
\text { of time. Such events may occur anytime during or after the end of the FUW } \\
\left(\mathrm{T}_{2}\right) \\
\text { Last recommended dosing event: treatment is lifelong, a date of prescription } \\
\text { discontinuation can only occur if treatment is switched (either within or after } \\
\text { the end of the FUW }\left(\mathrm{T}_{3}\right)\end{array}$ \\
\hline Objecti & $\begin{array}{l}\text { The intervention takes place within a University Hospital Transplantation } \\
\text { Center and consists of } 4 \text { consultations by a specialized nurse within } 6 \text { months. } \\
\text { We are interested in the intervention impact on adherence compared to a } \\
\text { control group receiving usual care, more specifically on implementation } \\
\text { during intervention and during follow-up compared to baseline. } \\
\text { Randomization will occur at between-patient level. Several patient-level } \\
\text { time-invariant (non-modifiable) characteristics were recorded and controlled }\end{array}$ \\
\hline
\end{tabular}




\begin{tabular}{|l|l|}
\hline & $\begin{array}{l}\text { for. Randomization was stratified by organ to account for differences in post- } \\
\text { transplant care models between services of the same center. Health system } \\
\text { level variables are considered constant. }\end{array}$ \\
\hline Sources: & $\begin{array}{l}\text { Participants can self-report behavior and socio-demographics (standardized } \\
\text { questionnaire at patient inclusion visit, and subsequent visits). At the time of } \\
\text { the study design, pharmacy refill data are not all collected electronically but } \\
\text { this may change in the near future. The prescription dates are currently } \\
\text { entered manually by pharmacy staff. Electronic monitoring is feasible due to } \\
\text { the clinical setting (regular visits to the same HCP organization for both usual } \\
\text { care and intervention groups), same mode of administration (oral) and dosing } \\
\text { regimen (one tablet every } 12 \pm 2 \text { hours). }\end{array}$ \\
\hline
\end{tabular}

Box 1: Operational definitions for measuring adherence in the example study

\section{Initiation: Not studied}

\section{Persistence: Not studied}

\section{Implementation:}

Quality of implementation measured by smart electronic monitoring device in relation to a) medication taking as missing a single or consecutive dose over a given period (between study visits) and b) medication timing as not taking tablets every 12 hours \pm 2 hours over 3 months. Quantification: sequences of daily binary scores per patient (correct/incorrect taking or timing) over each of the three periods (3-month baseline, 6-month intervention, 6-month follow-up); used to compare intervention and control groups over these 3 study periods using generalized estimating equations (GEE).

Summary measure: for descriptive purposes - mean daily proportions of patients with correct dosing/timing adherence (baseline; intervention; follow-up), from binary variables summarized per day across patients (percentage of patients with correct implementation), then averaged over each study period. 


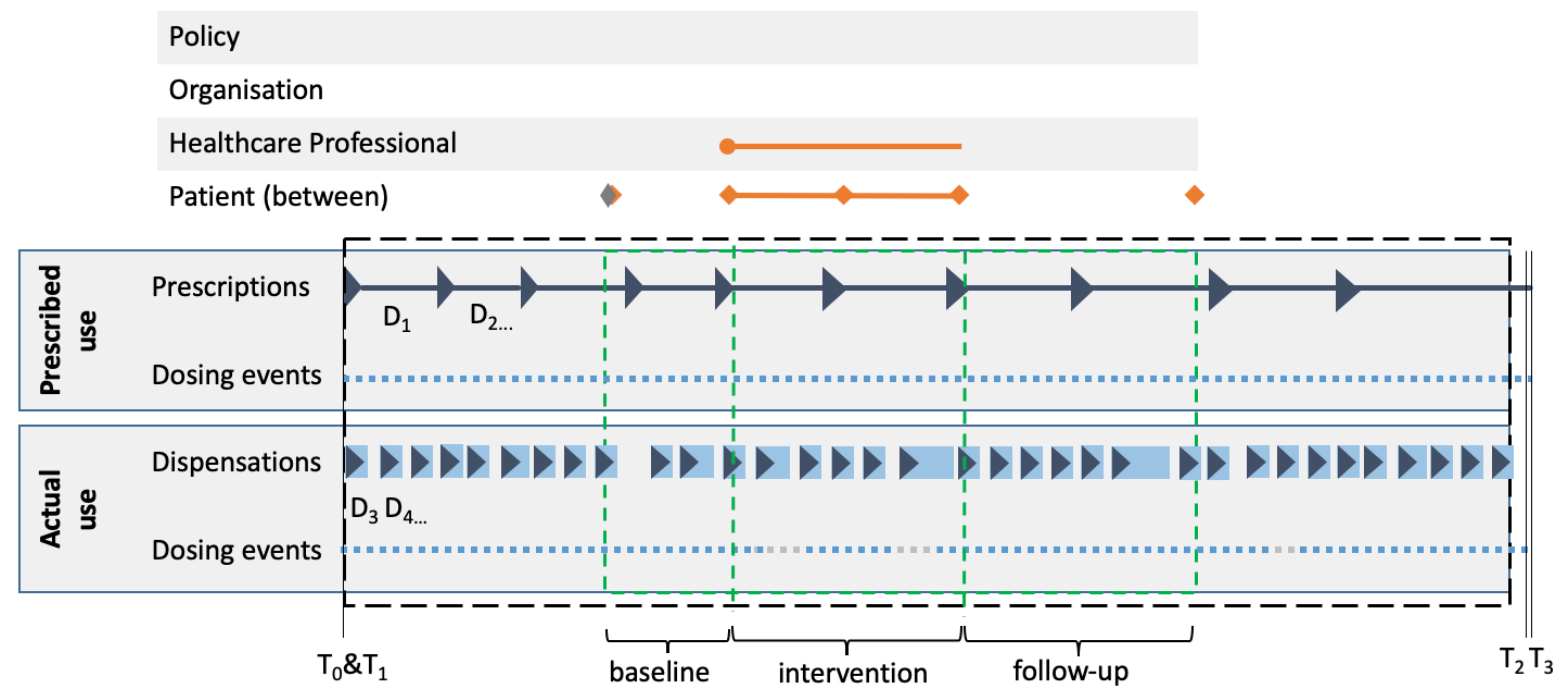

Figure 2: Example for a graphical representation of the TEOS exercise. $D_{1}-D_{4}$ and $T_{0}-T_{3}$ refer to the timelines and events, respectively, mentioned in Table 2 above. Dashed rectangles: Follow-up- window (black) and observation window (green). The intervention (orange line) is provided by nurses (HCP) to patients; adherence data are collected from patients at 5 moments (orange diamonds). Additional predictors and outcomes are measured at the patient level (grey lozenges).

References:

1. Dima AL, van Ganse E, Stadler G, de Bruin M. Does adherence to inhaled corticosteroids predict asthma-related outcomes over time? A cohort study. Eur Respir J. 2019:1900901. doi:10.1183/13993003.00901-2019

2. Dima AL, van Ganse E, Laforest L, Texier N, de Bruin M. Measuring medication adherence in asthma: Development of a novel self-report tool. Psychol Health. 2017;32:10:12881307. doi:10.1080/08870446.2017.1290248

3. Dobbels F, Bleser LD, Berben L, et al. Efficacy of a medication adherence enhancing intervention in transplantation: The MAESTRO-Tx trial. J Heart Lung Transplant. 2017;36(5):499-508. doi:10.1016/j.healun.2017.01.007 